an amputation had been performed or not despite a primary successful procedure.

Results: In this cohort of 19 patients, 16 were male (84\%), all were tobacco active users, and $21 \%$ cannabis users. Median follow-up was 19.9 months [11.0-36.9]. lloprost infusion had been tested for $84.2 \%$ of the patients before revascularisation, and $63.2 \%$ had a dihydropiridine, $73.3 \%$ a statin and $100 \%$ at least aspirin. Endovascular procedure was exclusive for 14 patients, and bypass surgery for 1 patient. Four patients had combined procedures. Globally, 44 procedures including 7 bypasses $(15.9 \%)$ and 37 endovascular surgeries $(84.1 \%)$, due to multiple procedures for some patients. Primary patency of endovascular procedures was $89.4 \%$ and their patency duration was $221 \pm 173$ days. For bypass surgery, the patency duration was higher $827 \pm 408$ days $(p=0.273$ ). Looking at the territory, aorto-iliac revascularizations gave better results on long term 1830 days followed by upper limb $751 \pm 402$ days, femoro-popliteal procedures $347 \pm 196$ days and finally infra-popliteal ones with $178 \pm 134$ days.

Stability or improvement was observed after $52.3 \%$ of the revascularizations. Among the failures, $21.2 \%$ were associated with a minor amputation, and $11.3 \%$ with a major amputation. No predicting factors of failure could have been determined.

Conclusions: We report the largest series of revascuarization in TAO. As expected, proximal artery revascularisation of lower limbs were associated with a higher and a longer patency rate than femoro-popliteal procedures and even more than infra-popliteal revascularisations. Suprinsingly, upper limb revascularisation had a better outcome than for lower limbs. Efficacy of revascularizations are difficult to assess because such procedures are often proposed as a salvage option. However, it is worth to note that more than $50 \%$ of the patients had a benefit from the revascularisation.

Disclosure of Interest: T. Mirault Grant/research support from: GENZYME (2010) M. Delahaye: None declared, A. Galloula: None declared, M. Guillet: None declared, S. Zarka: None declared, M.-C. Courtois: None declared, M. Sapoval: None declared, G. Goudot: None declared, E. Messas: None declared

DOI: 10.1136/annrheumdis-2018-eular.2169

\section{THU0468 SERUM ANGIOGENESIS BIOMARKERS PREDICT DISEASE OUTCOME IN GIANT CELL ARTERITIS}

Y. van Sleen, M. Sandovici, W. Abdulahad, J. Bijzet, A. Boots, E. Brouwer. Rheumatology and Clinical Immunology, University Medical Center Groningen, Groningen, Netherlands

Background: Giant cell arteritis (GCA) is characterised by inflammation of the medium and large vessels. Two forms of GCA are described, C(cranial)-GCA and LV(large vessel)-GCA, which can be present either separately or co-exist in a patient. Clinical features, erythrocyte sedimentation rate (ESR) and C-reactive protein (CRP) levels are used for disease diagnosis and monitoring, but are not disease specific. Relapses are common during and after treatment, therefore new biomarkers are needed for diagnosis and disease course prediction.

Objectives: As the majority of infiltrating cells in in the vessels of GCA patients are macrophages the aim of this project was to identify and compare levels of macrophage products in the serum of GCA patients as potential biomarkers.

Methods: Forty-one newly diagnosed GCA patients (temporal artery biopsy proven C-GCA and FDG-PET scan-positive LV-GCA) were recruited before start of glucocorticoids. Disease course was monitored and time to glucocorticoid treatment free remission was documented. Thirty age- and sex matched healthy controls (HCs) and 13 infection controls (bladder or lung infection) were also included. Serum concentrations of interleukin (IL)-6, serum amyloid A (SAA), soluble CD163 (sCD163), calprotectin, YKL-40 (human cartilage glycoprotein-39), vascular endothelial growth factor (VEGF), and angiopoietin-1 and 2 were determined by ELISA or Luminex assay.

Results: IL-6, SAA, SCD163, calprotectin, YKL-40, VEGF and angiopoietin-2 levels are increased in GCA and infection controls compared to HCs. IL-6 levels correlated strongly with $C R P, E S R$ and SAA, all markers of the Acute Phase Response (APR), in GCA. Interestingly, YKL-40, angiopoietin-2 and calprotectin levels showed only weak or no correlation with APR biomarkers, while they were strongly correlated with the APR in infection controls. Monocytes in peripheral blood correlated with APR biomarkers in GCA, whereas neutrophils correlated with the APR in infection controls. Patients with overlapping C-GCA and LV-GCA displayed a significantly stronger APR than patients with C-GCA or LV-GCA alone. High VEGF and angiopoietin-1, but low angiopoietin-2 levels at baseline predicted a shorter time to treatment free remission. This is in contrast to markers of the APR, which did not significantly predict time to treatment free remission. Conclusions: In this study, we show that markers of angiogenesis are better predictors of disease outcome than APR biomarkers. It appears that levels of calprotectin, YKL40 and angiopoietin-2 are increased through other than APR pathways during GCA compared to acute infection. Monocytes rather than neutrophils appear to drive the APR in GCA. This response is stronger in GCA patients with both cranial and systemic symptoms.
Disclosure of Interest: None declared

DOI: 10.1136/annrheumdis-2018-eular.6555

\section{THURSDAY, 14 JUNE 2018}

Osteoporosis

\section{THU0469 QUANTIFYING THE TREATMENT WITH GLUCOCORTICOIDS AS A RISK FACTOR FOR THE OCCURRENCE OF OSTEOPOROSIS AND FRACTURES IN PATIENTS WITH RA}

E. Wiebe, R. Biesen, K.N. Zeiner, D.C. Freier, S. Hermann, G.-R. Burmester,

F. Buttgereit. Department of Rheumatology and Clinical Immunology, Charité -

University Medicine Berlin, Berlin, Germany

Background: Rheumatoid arthritis (RA) is associated with increased systemic bone loss, leading to a high risk for fragility fractures. The etiology of increased fracture risk in RA is multifactorial and comprises next to general risk factors also RA-specific risks, most prominently chronic inflammation, seropositivity and glucocorticoid (GC) use ${ }^{1}$. Yet, there is evidence that GCs may, by adequately suppressing systemic inflammation, also have a positive effect on BMD and fracture risk in $R^{2}$.

Objectives: The purpose of this study was to investigate the prevalence of osteoporosis and fragility fractures in RA patients and to characterise, among other risk factors, the role of GC dose, cumulative dose (GCCD) and duration as well DMARD treatment on bone health.

Methods: Rh-GIOP is an ongoing prospective observational study collecting and analysing disease- and bone-related data from patients with chronic rheumatic diseases treated with GCs. In this cross-sectional analysis, we evaluated the initial visit of 238 patients with RA. Descriptive analyses were performed, with values displayed as mean/standard deviation and median/range for continuous variables. For subgroup analyses, non-parametric tests were used.

Results: Of 238 patients with RA ( $79.4 \%$ women, mean age: $63.6 \pm 12.5$ years) 155 were seropositive and 83 seronegative. Seronegative patients were numerically older (66.8 \pm 12.1 vs $61.8 \pm 12.1$ years) and more often in menopause $(78.3 \%$ vs $61.8 \%$, ns) than seropositive, while the latter had longer disease duration (median: 4.0 vs 11.0 years, $p=0.03$ ). Overall, osteoporotic BMD was more frequent at femoral sites, with $21 \%$ of patients having T-Scores $<-2.5$. Osteoporotic BMD was more common in seronegative patients (ns), although no difference in the frequency of fragility fractures ( $n=18 ; 24.1 \%$ vs $n=28 ; 23.2 \%$ ) was found. All patients received GCs (mean dose: $5.0 \pm 6.8 \mathrm{mg}$, mean GCCD $15.1 \pm 19.3 \mathrm{~g}$ mean duration $7.7 \pm 8.2$ years) with seropositive patients having numerically higher GCCD, longer duration of GC therapy and more often current GC doses above $>10 \mathrm{~g} /$ day. Biological DMARDs were more frequently used in seropositive patients $(n=20 ; 24.1 \%$ vs $n=67 ; 43.2 \%, p=0.02)$. Anti-osteoporotic therapies between both groups did not differ.

Neither current GC doses nor GCCD nor DMARD therapy had a statistically significant and independent effect on BMD or fragility fractures in either RA group.

Conclusions: Osteoporosis and fragility fractures remain a challenge in the management of RA, being determined by multiple interacting factors. Our data confirm that GCs may not per se increase fracture risk and decrease BMD in RA but rather, that optimal management of disease activity with or without GCs may be beneficial to bone health. Interestingly however, despite higher cumulative GC doses and duration, seropositive RA patients did not have lower BMD or higher prevalence of fragility fractures compared to seronegative patients. Further prospective data is warranted to better characterise the role of GCs and DMARDs in regard to osteoporosis and fracture risk in RA patients.

\section{REFERENCES}

[1] Briot K, et al. "Inflammatory Diseases and Bone Fragility." Osteoporosis International 2017 December 1;28(12):3301-14.

[2] Güler-Yüksel, et al. "Glucocorticoids, Inflammation and Bone." Calcified Tissue International 2018 January 8.

Disclosure of Interest: E. Wiebe Grant/research support from: Rh-GIOP is supported by a joint funding of Amgen, BMS, Celgene, Generic Assays, GSK, Horizon, medac, Mundipharma, Pfizer and Roche., R. Biesen Grant/research support from: Rh-GIOP is supported by a joint funding of Amgen, BMS, Celgene, Generic Assays, GSK, Horizon, medac, Mundipharma, Pfizer and Roche., K. Zeiner Grant/research support from: Rh-GIOP is supported by a joint funding of Amgen, BMS, Celgene, Generic Assays, GSK, Horizon, medac, Mundipharma, Pfizer and Roche., D. Freier Grant/research support from: Rh-GIOP is supported by a joint funding of Amgen, BMS, Celgene, Generic Assays, GSK, Horizon, medac, Mundipharma, Pfizer and Roche., S. Hermann: None declared, G.-R. Burmester: None declared, F. Buttgereit Grant/research support from: Rh-GIOP is supported by a 
joint funding of Amgen, BMS, Celgene, Generic Assays, GSK, Horizon, medac, Mundipharma, Pfizer and Roche.

DOI: 10.1136/annrheumdis-2018-eular.2732

\section{THU0470 NON-CLONAL ELEVATION OF SERUM IMMUNOGLOBULIN FREE LIGHT CHAINS IS PREDICTIVE OF HIP FRACTURE IN BOTH WOMEN AND MEN}

S. Amin, E.J. Atkinson, S.J. Achenbach, A. Dispenzieri, R.A. Kyle, S.V. Rajkumar. MAYO CLINIC, Rochester, USA

Background: Proinflammatory cytokines favour uncoupling of bone turnover and decreased bone density and strength, leading to increased fracture risk. Non-clonal elevation of serum immunoglobulin free light chains (sum of kappa and lambda chains) ( $\sum$ FLC) may be a global marker of generalised immune stimulation, and has been associated with chronic co-morbidities ${ }^{1}$ as well as increased mortality ${ }^{2}$. Objectives: We examined whether elevated $\sum F L C$ is associated with an increased risk for hip fractures in a population-based cohort.

Methods: We studied Olmsted County, Minnesota, USA residents, age $>50$ years, in whom $\sum$ FLC was measured between March 1995 and November 2003 and research authorisation was available. Anyone with a known plasma cell disorder was excluded. Using the Rochester Epidemiology Project, a unique medical records linkage system that allows access to all (inpatient and outpatient) community medical records for Olmsted County residents, we identified all hip fractures that occurred in subjects following their $\sum F L C$ measurement to their last available follow-up or the end of 2015. All available medical records were reviewed by trained nurse abstractors to validate hip fractures identified and to determine their antecedent cause (pathological process [e.g., malignancy], severe trauma [e.g., motor vehicle accidents] and those due to no more than moderate trauma [by convention, equivalent to a fall from standing height or less]) Charlson comorbidity index $(\mathbf{C C l})$ was determined at the time of baseline $\sum$ FLC measurement. We used a Cox proportional hazards model, stratified by sex, adjusting for age, serum creatinine and $\mathrm{CCl}$, to examine whether a $\sum F L C \geq 4.72 \mathrm{mg} / \mathrm{dl}$ (levels previously associated with increased mortality in this population ${ }^{1}$ ) is associated with an increased risk for hip fracture.

Results: We studied 15814 residents [mean age (SD), $64^{10} \mathrm{yrs} ; 8722$ women, 7092 men] of whom 796 (9.1\%) women and 781 (11.0\%) men had a $\sum F L C \geq 4.72 \mathrm{mg} / \mathrm{dl}$. Women and men with an elevated $\sum \mathrm{FLC} \geq 4.72 \mathrm{mg} / \mathrm{dl} \mathrm{had}$ higher CCI [median (IQR) $2(0,4)$ vs $0(0,2)$; same results for both sexes]. We identified 687 women and 255 men with a hip fracture from any cause (628 women and 220 men had a moderate trauma hip fracture), over 112171 person-years (p-y) and 89269 p-y of follow-up, respectively. We found that both women and men with $\sum F L C \geq 4.72 \mathrm{mg} / \mathrm{dl}$ had an increased risk for hip fracture due to any cause: hazard ratio $(\mathrm{HR})[95 \% \mathrm{Cl}] 1.31[1.00,1.73]$ in women, HR $1.97[1.37$, 2.83] in men; as well as for moderate trauma hip fractures: $\operatorname{HR} 1.39[1.05,1.84]$ in women, HR 2.12 [1.46, 3.09] in men.

Conclusions: We found that $\sum F L C \geq 4.72 \mathrm{mg} / \mathrm{dl}$ is associated with an increased risk for hip fracture in both women and men, independent of age and chronic comorbidities. Elevated $\sum$ FLC may be a marker of proinflammatory cytokines detrimental to bone health and warrants further study.

\section{REFERENCES:}

[1] Nakano T, et al. Free Immunoglobulin Light Chain: Its Biology and Implications in Diseases. Clinica Chimica Acta. 2011;412(11-12):843-9.

[2] Dispenzieri A, et al. Use of Nonclonal Serum Immunoglobulin Free Light Chains to Predict Overall Survival in the General Population. Mayo Clinic Proceedings 2012;87(6):517-523.

Acknowledgements: Supported by grant P01 AG04875, R01 CA107476 and R01 AG034676 from the National Institutes of Health, U.S. Public Health Service. Disclosure of Interest: None declared

DOI: 10.1136/annrheumdis-2018-eular.6041

\section{THU0471 RISK FACTORS OF LOW BONE MINERAL DENSITY IN PATIENTS WITH SYSTEMIC SCLEROSIS}

S.V. Monov ${ }^{1}$, D. Monova ${ }^{2}$, M. Stambolova ${ }^{3}$, R. Shumnalieva ${ }^{1} .{ }^{1}$ Department of Rheumatic Diseases, Medical University - Sofia; ${ }^{2}$ Department of Internal Medicine, Medical University - Sofia, Medical Institute; ${ }^{3}$ Department of Internal Medicine, Medical Institute - Ministry of Interior, Sofia, Bulgaria

Background: Systemic sclerosis (SSc) is a multisystem autoimmune disease, characterised by diffuse fibrosis, degenerative changes, and vascular abnormalities in the skin, joints, and internal organs. The effect of systemic sclerosis on bone density is not well understood.

Objectives: The aim of this study is to evaluate the risk factors of low bone mineral density (BMD) and occurrence of fracture and fracture-related mortality in patients with SSc.

Methods: Demographics, disease manifestations of SSc, biological inflammatory parameters, functional disability, scleroderma health assessment questionnaire immunological status, BMD (lumbar spine and femoral neck), risk factors for low $\mathrm{BMD}$, fractures, and fracture-related mortality were collected in patients with SSc. BMD was measured by using a dual-energy $X$-ray absorptiometry in lumbar spine (L1-L4) and femoral neck. Fisher's Exact and Student's t-tests were used to evaluate differences between women with and without low BMD. Logistic regression was used for multivariate analysis.

Results: Forty eight consecutive unselected SSc women were approached. The mean age of women was $48,32 \pm 15,56$ years, the mean disease duration was $10,12 \pm 4,78$ years, Twenty-nine women $(60,42 \%)$ had low BMD, of those 11 $(37,93 \%)$ had osteoporosis, mean BMD in lumbar spine was $-2,86 \pm 0,38$ and in femoral neck was $-2,22 \pm 0,26$. Twenty three $(47,92 \%)$ women with SSc were postmenopausal. In correlation analysis and in multiple regression models, there were correlations between BMD and longer duration of SSc $(p<0,01)$, family his tory of osteoporosis $(p<0.05)$, age $(p<0,01)$, menopause $(p<0,01)$, low body mass index $(p<0,02)$, presence of internal organ involvement $(p<0,05)$, malabsorption syndrome $(p<0,05)$, joint involvement (severe joint pain and erosive arthropathy) $(p<0,05)$, and immunological status (positivity of anti-DNA topoisomerase I antibodies) $(p<0,05) .4$ women $(4 / 29)$ with low BMD had a fracture, compared to 2 without low BMD. Fracture-related mortality did not occur in any patients.

Conclusions: Our data suggest that women with SSc are at risk of low BMD and fracture, especially when other risk factors for osteoporosis are present. A number of clinically relevant factors (longer duration of SSc, presence of internal organ involvement, joint involvement, immunological status) are associated with low BMD.

Disclosure of Interest: None declared

DOI: 10.1136/annrheumdis-2018-eular.1886

\section{THU0472 FACTORS ASSOCIATED WITH READINESS FOR ADOPTING OSTEOPOROSIS TREATMENT CHANGE}

M.I. Danila ${ }^{1}$, E.J. Rahn ${ }^{1}$, A.S. Mudano ${ }^{1}$, R.C. Outman ${ }^{1}$, P. Li ${ }^{1}$, D.T. Redden ${ }^{1}$, F. A. Anderson ${ }^{2}$, S.L. Greenspan ${ }^{3}$, A.Z. LaCroix ${ }^{4}$, J.W. Nieves ${ }^{5}$, S.L. Silverman ${ }^{6}$, E. S. Siris ${ }^{7}$, N.B. Watts ${ }^{8}$, S. Ladores ${ }^{1}$, K.M. Meneses ${ }^{1}$, J.R. Curtis ${ }^{1}$, K.G. Saag ${ }^{1}$ ${ }^{1}$ University of Alabama at Birmingham, Birmingham; ${ }^{2}$ University of Massachusetts Medical School, Worcester, ${ }^{3}$ University of Pittsburgh, Pittsburgh; ${ }^{4}$ Group Health Cooperative, Seattle; ${ }^{5}$ Helen Hayes Hospital, West Haverstraw, ${ }^{6}$ Cedars-Sinai Medical Center, Los Angeles; ${ }^{7}$ Columbia University Medical Center, New York; ${ }^{8}$ Mercy Health Osteoporosis and Bone Health Services, Cincinnati, USA

Background: Understanding factors associated with the readiness for adopting osteoporosis treatment change may inform the design of behavioural interventions to improve osteoporosis treatment uptake in women at high risk for fracture. Objectives: To examine the factors associated with the readiness for adopting osteoporosis treament change among US women with prior fractures.

Methods: US women in the Global Longitudinal Study of Osteoporosis (GLOW) with prior self-reported fractures who were not currently using osteoporosis therapy were eligible to participate in the Activating Patients at Risk for OsteoPOroSis (APROPOS) Study. Participants' readiness for behaviour change was assessed using a modified form of the Weinstein Precaution Adoption Process Model (PAPM). We defined pre-contemplative participants as those who self-classified in the unaware and unengaged stages of PAPM. Contemplative participants were defined by the undecided, decided not to act, and decided to act stages of PAPM. Bivariate tests and stepwise multivariable logistic regression evaluated the following factors associated with these two levels of readiness for behaviour change: sociodemographic characteristics, health literacy, self-reported history of depression and dementia, previous treatment for osteoporosis, whether participants had been told they had osteoporosis/osteopenia, and whether they had concerns about osteoporosis.

Results: A total of 2684 women were enrolled in APROPOS. Participants were $95 \%$ Caucasian, with a mean (SD) age $74.9(8.0)$ years and $77 \%$ had some college education. Overall, $25 \%(n=544)$ self-classified in the contemplative stage of behaviour change. Compared to women who self-classified as pre-contemplative, contemplative women were more likely to be concerned about osteoporosis (adjusted OR $[\mathrm{aOR}]=3.2,95 \% \mathrm{Cl} 2.3-4.4$ ) and to report prior osteoporosis treatment (aOR 4.3, 95\% Cl 3.1-6.0). Participants who were told they had osteoporosis had a 12.4 fold odds to be in the contemplative group (95\% $\mathrm{Cl} 8.5-18.1$ ), while those who were told they had osteopenia had 4.1 fold odds to be in the contemplative group $(95 \% \mathrm{Cl} 2.9-5.9)$. 